Supplement of

\title{
Versatile soil gas concentration and isotope monitoring: optimization and integration of novel soil gas probes with online trace gas detection
}

Juliana Gil-Loaiza ${ }^{1}$, Joseph R. Roscioli², Joanne H. Shorter ${ }^{2}$, Till H.M. Volkmann ${ }^{3,4}$, Wei-Ren Ng ${ }^{3}$, Jordan E. Krechmer ${ }^{2}$, Laura K. Meredith ${ }^{1,3, *}$

${ }^{1}$ School of Natural Resources and the Environment, University of Arizona, Tucson, AZ, 85721, USA

${ }^{2}$ Aerodyne Research Inc., Billerica, MA, 01821, USA

${ }^{3}$ Biosphere 2, University of Arizona, Oracle, AZ, 85623, USA

${ }^{4}$ Applied Intelligence, Accenture, Kronberg im Taunus, Hesse, 61476, Germany.

Correspondence to: Laura K. Meredith laurameredith@email.arizona.edu

\section{Contents of this file}

Figures S1 to S4

Tables S1 to S3

References 


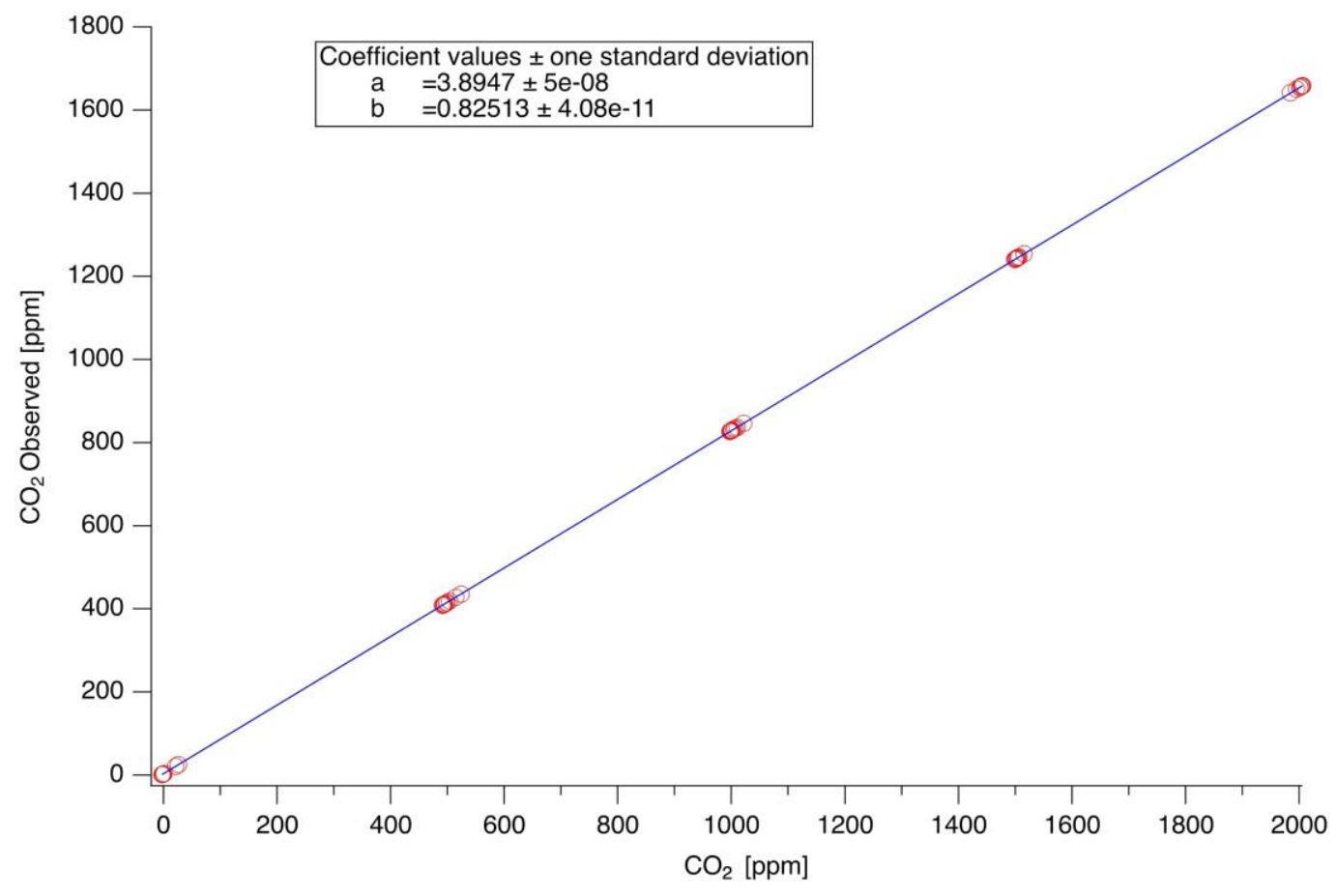

Figure S1. Calibration of absolute concentration of $\mathrm{CO}_{2}$ in silica matrix (System 1 Dual) between observed and controlled dilution mixtures using linear regression relationship

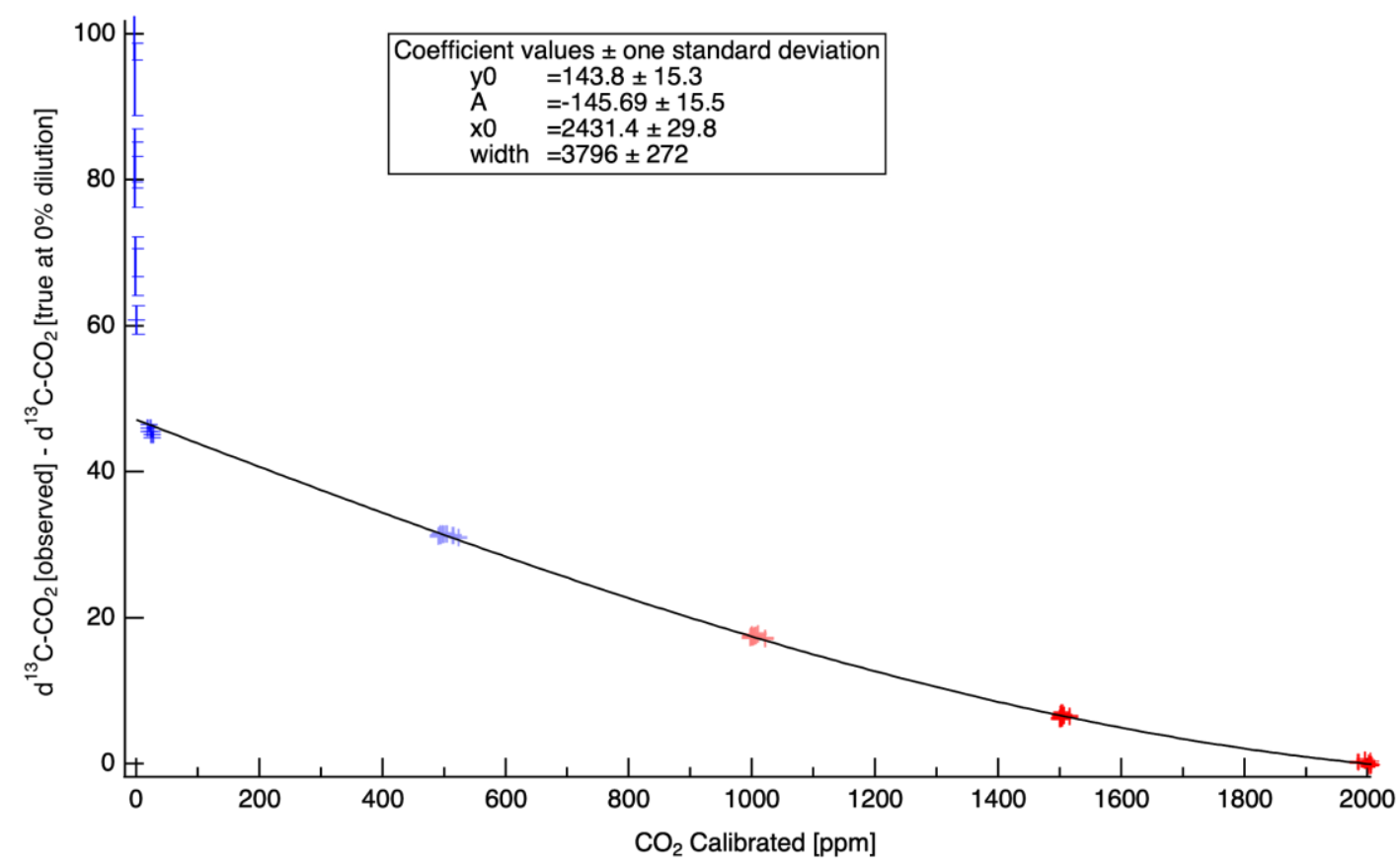

Figure S2. $\quad \Delta^{13} \mathrm{C}-\mathrm{CO}_{2}$ calibration from the concentration dependent relationship of dual $\delta^{13} \mathrm{C}-\mathrm{CO}_{2}$ vs observed $\left[\mathrm{CO}_{2}\right]$. The Gaussian equation was used to fit the relationship between $\left(\delta^{13} \mathrm{C}-\mathrm{CO}_{2}\right.$ observed $-\delta^{13} \mathrm{C}_{-} \mathrm{CO}_{2}$ true and $\mathrm{CO}_{2}$ concentration. $\delta^{13} \mathrm{C}-$ $\mathrm{CO}_{2}$ calibration offset calibration curve was used to correct observations. 
(a)
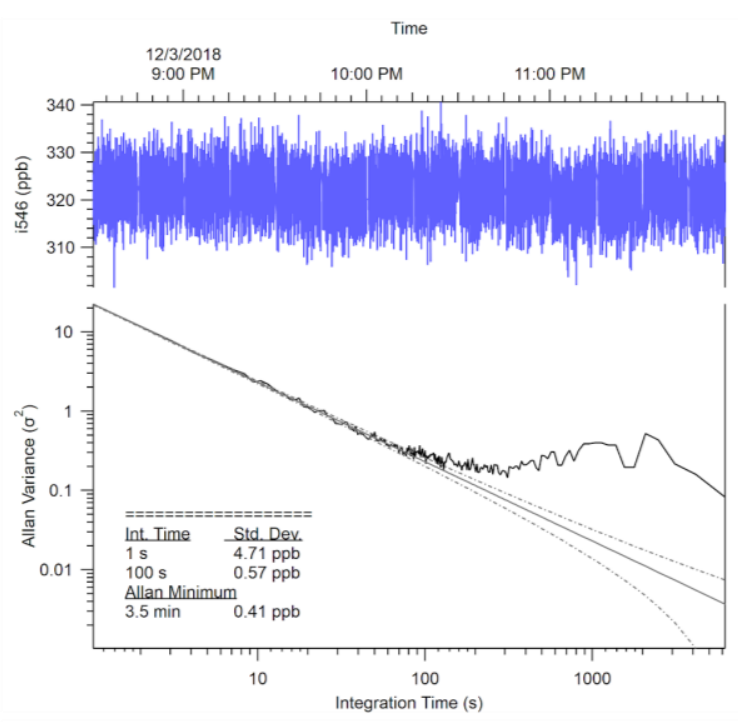

(c)

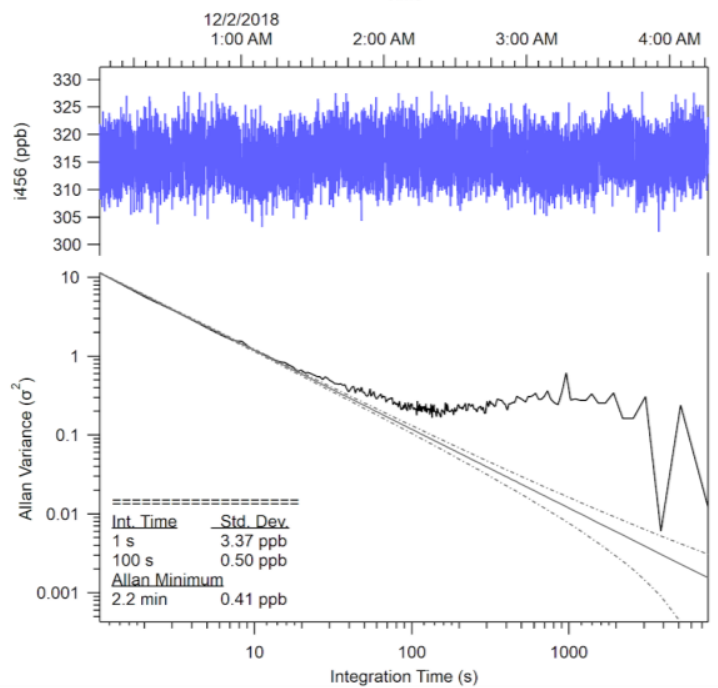

(b)
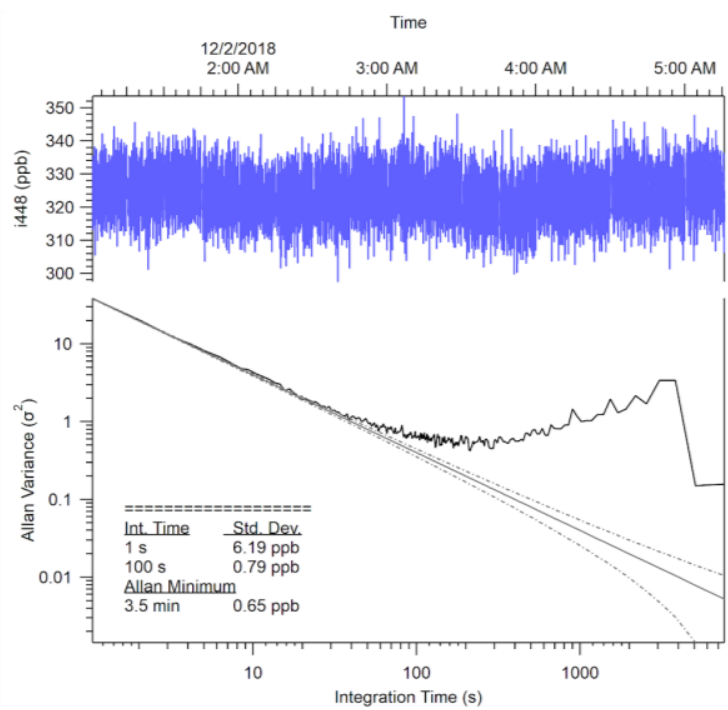

(d)
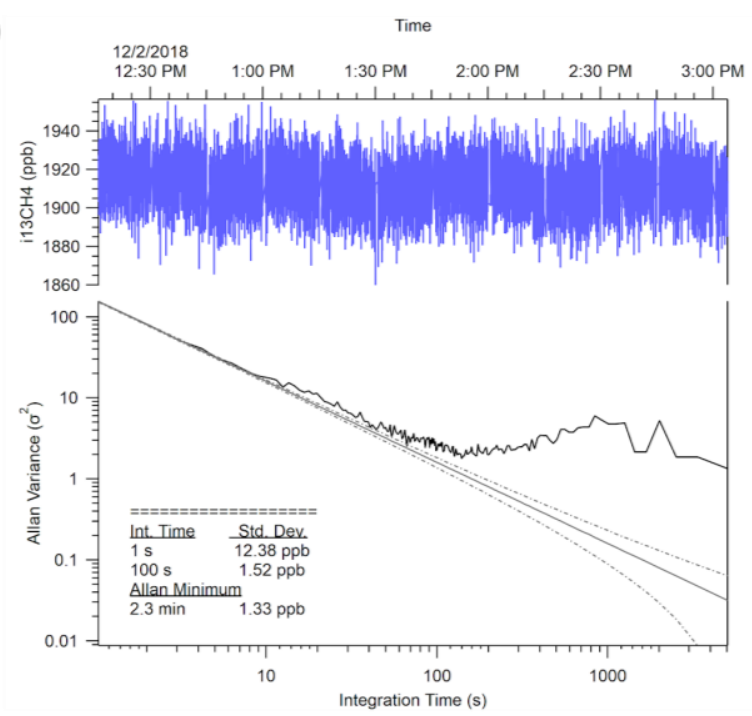

Figure S3. Allan-Werle plots of precision for individual minor isotopes $\mathrm{N}_{2} \mathrm{O} 456$ and 546 (top), 448 and ${ }^{13} \mathrm{C}-\mathrm{CH}_{4}$ (bottom). Precision for $\square 456=1.3$ per mil, $\square 456=1.4$ permil, $\square 448=2.2$ per mil, and $\square{ }^{13} \mathrm{C}=0.7$ per mil 


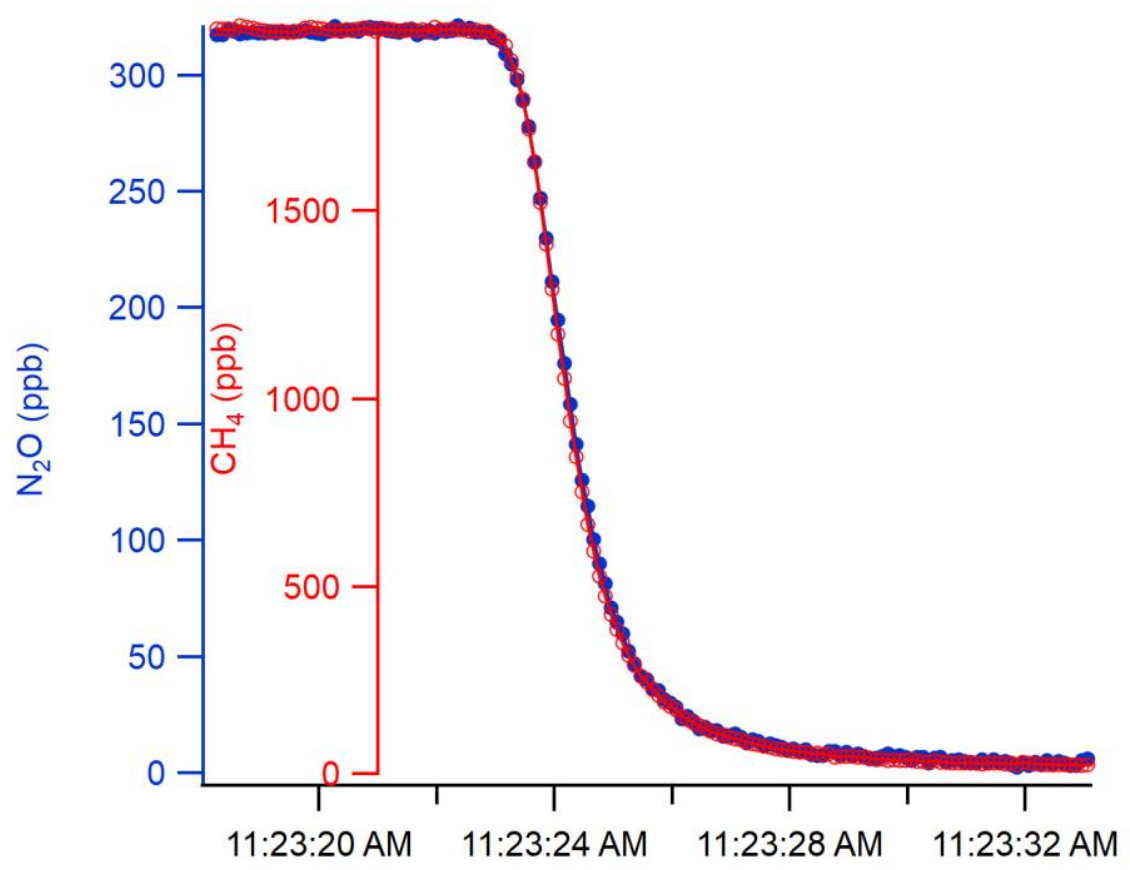

Figure S4. Improvement in the time response with the addition of the insert. Time Response of 76m standard cell with insert. $\mathrm{P}=30$ Torr, flow 1 slpm.

Table S1. Soil matrix description and particle size distribution

\begin{tabular}{|c|c|c|c|c|}
\hline $\begin{array}{l}\text { Type of } \\
\text { Matrix }\end{array}$ & Soil ID & Vendor & Particle size distribution (units) & Type of soil \\
\hline $\begin{array}{l}\text { Granusil } \\
4095^{1}\end{array}$ & Silica & $\begin{array}{l}\text { Covia } \\
\text { Corporation }\end{array}$ & $\begin{array}{l}8(0 \%), 16(0.28 \%), 20(27.66 \%), 30(52.55 \%), \\
40(15.99 \%), 50(3.22 \%), 70(0.22 \%), 100 \\
(0.04 \%), 140(0.02 \%), 200(0.28 \%)^{\mathrm{a}}\end{array}$ & $\begin{array}{l}\text { High purity industrial } \\
\text { quartz, Hardness (Mohs) } \\
7.0, \text { Moisture content } \\
<0.1 \% \text {. }\end{array}$ \\
\hline Type of Soil & Soil ID & Source Site & Sampling Location & Properties \\
\hline $\begin{array}{l}\text { Tropical } \\
\text { Rainforest }\end{array}$ & Soil 1 & \multirow{2}{*}{$\begin{array}{l}\text { Biosphere } 2 \\
\text { Tropical } \\
\text { Rainforest }\end{array}$} & Shaded Lowland (dieffenbachia, vine) & \multirow{2}{*}{$\begin{array}{l}\text { Sandy, silty loam (40-70\% } \\
\text { sand } 36 \% \text { silt and } 30 \% \\
\text { clay) }{ }^{\text {b; }} \text { and C:N 8.75; pH } \\
7-8^{\text {c }}\end{array}$} \\
\hline $\begin{array}{l}\text { Tropical } \\
\text { Rainforest }\end{array}$ & Soil 3 & & South facing terrace (ginger, hibiscus) & \\
\hline
\end{tabular}

${ }^{\text {a }}$ Mesh units ASTM; ${ }^{\mathrm{b}}$ (Lin et al., 1999) in (Smith et al., 2020); ${ }^{\mathrm{c}}$ (Van Haren et al., 2005)

Table S2. Measurement precision of the $76 \mathrm{~m}$ dual TILDAS for $\mathrm{N}_{2} \mathrm{O} / \mathrm{CH}_{4}$ isotopes 


\begin{tabular}{|l|c|c|}
\hline & $\begin{array}{c}\text { Mixing Ratio } \\
(\mathbf{p p b})\end{array}$ & $\square(\%)$ \\
\hline $\begin{array}{l}\mathbf{\delta 5 4 6} \mathbf{N}_{\mathbf{2}} \mathbf{O} \\
\left({ }^{15} \mathrm{~N}^{14} \mathrm{~N}^{16} \mathrm{O}\right)\end{array}$ & 0.46 & 1.4 \\
\hline $\begin{array}{l}\mathbf{8 4 5 6} \quad \mathbf{N}_{2} \mathbf{O} \\
\left({ }^{14} \mathrm{~N}^{15} \mathrm{~N}^{16} \mathrm{O}\right)\end{array}$ & 0.42 & 1.3 \\
\hline $\begin{array}{l}\mathbf{\delta 4 4 8} \quad \mathbf{N}_{2} \mathbf{O} \\
\left({ }^{14} \mathrm{~N}^{14} \mathrm{~N}^{18} \mathrm{O}\right)\end{array}$ & 0.72 & 2.2 \\
\hline${ }^{13} \mathbf{C}-\mathbf{C H}_{4}$ & 1.3 & 0.7 \\
\hline
\end{tabular}

Table S3. References used to estimate the 3D map for $\mathrm{N}_{2} \mathrm{O}$ isotopic signatures of bulk $\delta^{15} \mathrm{~N}$ (x-axis), $\delta^{18} \mathrm{O}$ (y-axis), and site preference (z-axis) (Figure 12b).

\begin{tabular}{|l|c|}
\hline \multicolumn{1}{|c|}{ Microbial activity } & Reference \\
\hline Bacterial Denitrification & $\begin{array}{r}\text { (Frame and Casciotti, 2010; Sutka et al., 2006; Toyoda } \\
\text { et al., 2005; Zou et al., 2014) }\end{array}$ \\
\hline Chemodenitrification & $\begin{array}{r}\text { (Jones et al., 2015; Toyoda et al., 2005; Wei et al., } \\
\text { Bacterial nitrification }\end{array}$ \\
\hline AOA & (Jung et al., 2014; Sutka et al., 2006; Yoshida, 1988) \\
\hline Fungal denitrification & (Sutka et al., 2015) \\
\hline
\end{tabular}

\section{References}

Frame, C. H. and Casciotti, K. L.: Biogeochemical controls and isotopic signatures of nitrous oxide production by a marine ammonia-oxidizing bacterium, Biogeosciences, 7, 2695-2709, 2010.

Hu, H.-W., Chen, D. and He, J.-Z.: Microbial regulation of terrestrial nitrous oxide formation: understanding the biological pathways for prediction of emission rates, FEMS Microbiol. Rev., 39(5), 729-749, 2015. 
Jones, L. C., Peters, B., Lezama Pacheco, J. S., Casciotti, K. L. and Fendorf, S.: Stable isotopes and iron oxide mineral products as markers of chemodenitrification, Environ. Sci. Technol., 49(6), 3444-3452, 2015.

Jung, M.-Y., Well, R., Min, D., Giesemann, A., Park, S.-J., Kim, J.-G., Kim, S.-J. and Rhee, S.-K.: Isotopic signatures of $\mathrm{N}_{2} \mathrm{O}$ produced by ammonia-oxidizing archaea from soils, ISME J., 8(5), 1115-1125, 2014.

Lin, G., Adams, J., Farnsworth, B., Wei, Y., Marino, B. D. V. and Berry, J. A.: Ecosystem carbon exchange in two terrestrial ecosystem mesocosms under changing atmospheric $\mathrm{CO}_{2}$ concentrations, Oecologia, 119(1), 97-108, 1999.

Smith, M. N., Taylor, T. C., van Haren, J., Rosolem, R., Restrepo-Coupe, N., Adams, J., Wu, J., de Oliveira, R. C., da Silva, R., de Araujo, A. C., de Camargo, P. B., Huxman, T. E. and Saleska, S. R.: Empirical evidence for resilience of tropical forest photosynthesis in a warmer world, Nat Plants, 6(10), 1225-1230, 2020.

Sutka, R. L., Ostrom, N. E., Ostrom, P. H., Breznak, J. A., Gandhi, H., Pitt, A. J. and Li, F.: Distinguishing nitrous oxide production from nitrification and denitrification on the basis of isotopomer abundances, Appl. Environ. Microbiol., 72(1), 638-644, 2006.

Sutka, R. L., Adams, G. C., Ostrom, N. E. and Ostrom, P. H.: Isotopologue fractionation during $\mathrm{N}_{2} \mathrm{O}$ production by fungal denitrification, Rapid Commun. Mass Spectrom., 22(24), 3989-3996, 2008.

Toyoda, S., Mutobe, H., Yamagishi, H., Yoshida, N. and Tanji, Y.: Fractionation of $\mathrm{N}_{2} \mathrm{O}$ isotopomers during production by denitrifier, Soil Biol. Biochem., 37(8), 1535-1545, 2005.

Van Haren, J. L. M., Handley, L. L., Biel, K. Y., Kudeyarov, V. N., McLain, J. E. T., Martens, D. A. and Colodner, D. C.: Drought-induced nitrous oxide flux dynamics in an enclosed tropical forest, Glob. Chang. Biol., 11(8), 12471257, 2005.

Wei, J., Zhou, M., Vereecken, H. and Brüggemann, N.: Large variability in CO2 and N2O emissions and in $15 \mathrm{~N}$ site preference of $\mathrm{N} 2 \mathrm{O}$ from reactions of nitrite with lignin and its derivatives at different $\mathrm{pH}$, Rapid Commun. Mass Spectrom., 31(16), 1333-1343, 2017.

Yoshida, N.: 15N-depleted N2O as a product of nitrification, Nature, 335(6190), 528-529, 1988.

Zou, Y., Hirono, Y., Yanai, Y., Hattori, S., Toyoda, S. and Yoshida, N.: Isotopomer analysis of nitrous oxide accumulated in soil cultivated with tea (Camellia sinensis) in Shizuoka, central Japan, Soil Biol. Biochem., 77, 276291, 2014. 